\title{
Traveltime inversion for 2-D anomaly structures
}

\author{
Danilo R. Velis ${ }^{1}$ \\ (Accepted in Geophysics, February 13, 2001)
}

\begin{abstract}
This work presents a traveltime inversion method that utilizes parametric functions for representing 2-D anomaly structures. These functions are described by a small set of unknown parameters which are in turn obtained after solving a highly nonlinear optimization problem via simulated annealing (SA). The procedure favors neither smooth nor high contrasting anomalies and keeps the number of unknowns very small so as to make the problem tractable using SA. Yet, the strategy allows one to accommodate a large class of velocity models. Results indicate that this new approach typically yields better images compared with a standard linearized inversion based on a cell parameterization scheme.
\end{abstract}

\section{Introduction}

Traveltime tomography is a valuable tool for imaging the Earth subsurface. Curved ray traveltime tomography was originally developed by Bois et al. (1972) for estimating the velocity distribution between two wells. As opposed to straight ray traveltime tomography, curved ray traveltime tomography is a highly nonlinear problem. This is due to the fact that the arrival-times are nonlinearly related to the unknown velocity field. In other words, not only the velocity distribution is unknown, but also the raypaths. Usually, the problem is solved using linearizing techniques in an iterative fashion, where the velocity field is represented by a network of cells with constant velocity. In general, a good starting model is required and some form of regularization or model constraints must be introduced in the objective function to stabilize the solution and to minimize the artifacts generated by the inversion, for the nonuniqueness associated with this underdetermined inverse problem (Nolet, 1987; Bregman et al., 1989). The main difficulty comes from the fact that usually the ray density in some regions of the grid is very low (Vesnaver, 1994).

The use of basis functions different from a rigid grid of rectangular cells offer an interesting approach to solve the tomography problem. In many cases, it is possible to obtain good fits to the true model and to inhibit the creation of inversion artifact using relative coarse meshes or appropriate parametric representations. Michelini (1995), for example, uses and adaptive B-spline gridding procedure where the node positions are optimized together with the velocity values. Velis and Ulrych (1995) carry out this optimization by means of SA. Sen et al. (1993) also combine B-splines and SA, but for the inversion of resistivity data. Natural pixels have been used by Michelena and Harris (1991) to also significantly reduce the number of degrees of freedom in traveltime tomography. Other strategies make use of adaptive irregular grids to increase the number of pixels in areas well constrained by the data (high ray density), and to decrease their number in poorly covered domains (Böhm et al., 1997; Böhm and Vesnaver, 1999).

In this work I use parametric functions for defining 2-D anomaly structures, either smooth or high contrasting, using a few adjustable parameters (Velis, 1998b; Velis, 1998a). The inverse problem is then cast as a nonlinear optimization problem where the unknowns are the center of the anomaly, size, shape, etc. These parameters, which are complemented by a background velocity model (e.g. a linear trend), are obtained by means of SA. The coefficients of the trend represent extra parameters in the traveltime inversion. Chunduru et al. (1995) also use SA to estimate the parameters defining 2-D geometrical bodies in a resistivity inversion context. But these structures are particular cases of the parameterization scheme adopted in this work to solve the tomography problem.

The parametric functions introduce strong nonlinearities into the optimization problem. Besides the resulting objective function (misfit between observed and calculated arrival times) is multimodal and rather ill-behaved. To avoid premature convergence and local minima, I make use of very fast simulated annealing (VFSA) (Ingber, 1989) to find the global minimum of the objective function. Traveltimes at each iteration are computed using a finite difference method (Vidale, 1988; Aldridge and Oldenburg, 1993). The strategy may find application in mining and archaeology prospection, as well as in other near surface studies.

\footnotetext{
${ }^{1}$ Departamento de Geofísica Aplicada, Observatorio Astronómico, Paseo del Bosque s/n, La Plata 1900, Argentina. E-mail: velis@fcaglp.unlp.edu.ar
} 

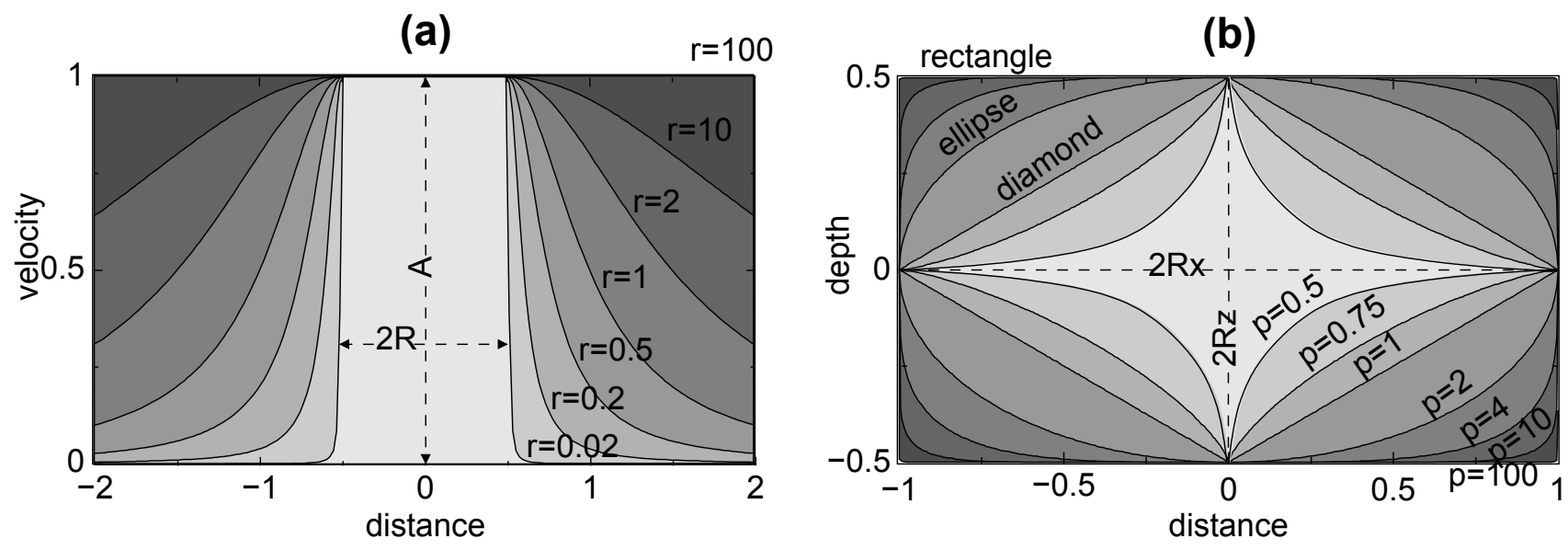

Figure 1: (a) One-dimensional velocity anomaly function for various slopes. (b) Two-dimensional velocity anomaly function for various shape parameters (flat region only).

\section{Model representation}

The parameterization scheme adopted in this paper for representing a 2-D velocity field, $v(x, z)$, is intended to offer an alternative to cell parameterization so as to reduce the number of parameters to a minimum. Yet, it allows a certain flexibility to accommodate complex structures.

I constructed 2-D anomalies based on 1-D functions. Essentially, the anomaly is represented by two separate parts: (1) a "flat" region of width $2 R$ and amplitude $A$; and (2) the "slopes" at each side of the flat region. Let $x=x_{c}$ be the center of the 1-D anomaly, then I define its velocity as

$$
v_{a}(x)= \begin{cases}A, & \left|x-x_{c}\right| \leq R \\ f\left(\left|x-x_{c}\right|-R\right), & \text { otherwise }\end{cases}
$$

where

$$
f(x)=\frac{A}{1+\left(\frac{x}{\rho R}\right)^{2}}, \quad \rho>0
$$

This function has its maximum, $A$, at $x=0$, and tends to zero asymptotically as $x \rightarrow \pm \infty$. The factor $\rho$, a dimensionless constant, is defined in such a way that $\rho R$ is the distance from the center at which the maximum of $f(x)$ decreases by a factor of two. That is, $f(|\rho R|)=f(0) / 2=A / 2$. This parameter is a measure of the width of the function $f(x)$. As a result, it controls the slopes of function $v_{a}(x)$.

Figure 1a shows a series of 1-D anomalies with unit amplitude and unit width, centered at $x_{c}=0$. Notice how the slopes of $v_{a}(x)$ vary with different values of $\rho$. For $\rho \rightarrow 0, f(x) \rightarrow 0$, and the anomaly reduces to a boxcar. For $\rho \rightarrow \infty, f(x) \rightarrow A$, and the "anomaly" is flat for all $x$. It is important to remark that equation (1) is continuous and differentiable everywhere, even at $x=x_{c} \pm R$, since $f(0)=A$, and $f^{\prime}(0)=0$. Equation (1) allows one to model either smooth or high contrasting 1-D anomalies, using only 4 parameters, namely $x_{c}, A, R$, and $\rho$.

In 2-D, one can make use of equation (1) to construct 2-D anomaly functions with similar features. Let's redefine the "radius" of the flat region, $R$, as the distance between the center of the anomaly, $\left(x_{c}, z_{c}\right)$, and the following curve:

$$
\frac{\left|x-x_{c}\right|^{p}}{R_{x}^{p}}+\frac{\left|z-z_{c}\right|^{p}}{R_{z}^{p}}=1, \quad p>0 .
$$

Here I have introduced a new parameter, $p$, that helps to control the shape of the flat region boundary. Notice that for $p=2$, equation (3) becomes an ellipse of center $\left(x_{c}, z_{c}\right)$ and semiaxes $R_{x}$ and $R_{z}$. Further, if $R_{x}=R_{z}$, it becomes a circle. In practice, $R$ is calculated by finding the intersection point, $\left(x_{R}, z_{R}\right)$, between the line connecting $\left(x_{c}, z_{c}\right)$ and $(x, z)$, with the curve $(3)$, where $(x, z)$ is a generic point in the $x z$-plane. After some algebraic manipulation,

$$
\left\{\begin{array}{l}
x_{R}=x_{c}+\frac{x-x_{c}}{\tilde{r}} \\
z_{R}=z_{c}+\frac{z-z_{c}}{\tilde{r}}
\end{array}\right.
$$


where

$$
\tilde{r}=\left(\frac{\left|x-x_{c}\right|^{p}}{R_{x}^{p}}+\frac{\left|z-z_{c}\right|^{p}}{R_{z}^{p}}\right)^{1 / p} .
$$

Then, since $R(x, z)=r\left(x_{R}, z_{R}\right), R=r / \tilde{r}$, where $r=\sqrt{ }\left[\left(x-x_{c}\right)^{2}+\left(z-z_{c}\right)^{2}\right]$

To define the slopes of the anomaly, it is useful to keep the same shape that is used for the 1-D case for $r>R$, that is

$$
f(r)=\frac{A}{1+\left(\frac{r}{\rho R}\right)^{2}}
$$

Finally, the 2-D anomaly function is defined as

$$
v_{a}(r)= \begin{cases}A, & r \leq R \\ f(r-R), & \text { otherwise }\end{cases}
$$

As in the 1-D case, $v_{a}(r)$ is continuous up to the first derivative, except for $p \leq 1$. The flexibility of this model representation and the meaning of all the parameters required to define the $2-\mathrm{D}$ anomaly, are illustrated in Figure $1 \mathrm{~b}$ for various $p,\left(x_{c}, z_{c}\right)=(0,0), R_{x}=1.0$, and $R_{z}=0.5$. Notice how the anomaly takes on different shapes as $p$ varies. The shape of the anomaly can be changed easily by adjusting the appropriate parameters for the flat region, along with the factor $\rho$. For example, values of $p$ smaller than or equal to one can also be selected. The resulting flat region takes on a diamond-like shape. For $p=2$ the flat region becomes an ellipse (or a circle), and for $p \rightarrow \infty$ it becomes a rectangle of size $2 R_{x} \times 2 R_{z}$.

In addition, the whole anomaly is rotated an angle $\theta$ around the axis normal to the $x z$-plane passing through its center. This is done using the transformation

$$
\left\{\begin{array}{l}
x^{\prime}=x_{c}+\left(x-x_{c}\right) \cos \theta-\left(z-z_{c}\right) \sin \theta \\
z^{\prime}=z_{c}+\left(x-x_{c}\right) \sin \theta+\left(z-z_{c}\right) \cos \theta
\end{array}\right.
$$

where $\left(x^{\prime}, z^{\prime}\right)$ are the new coordinates in the rotated frame. Finally, a linear background can also be included:

$$
v_{b}(x, z)=v_{0}+g_{x}\left(x-x_{\min }\right)+g_{z}\left(z-z_{\min }\right),
$$

where $v_{0}$ is the background velocity, and $g_{x}$ and $g_{z}$ are the velocity gradients in each dimension. The total number of parameters is $K=11$. That is, number of anomaly parameters + number of background coefficients. Then, the model space can be expressed as the $K$-length vector given by

$$
\mathbf{m}=\left\{A, x_{c}, z_{c}, R_{x}, R_{z}, p, \rho, \theta, v_{0}, g_{x}, g_{z}\right\}
$$

The described strategy for representing 2-D anomaly structures can be readily extended for dealing with 3-D models. In this case one can use the same formulation as in the 2-D case, with the addition of extra parameters such as $y_{c}$, $R_{y}, \xi$, and $g_{y}$, for the center, scale, azimuth, and velocity gradient, respectively.

\section{Forward modeling}

I adopted the finite-difference (FD) method (Vidale, 1988; Aldridge and Oldenburg, 1993) to compute the traveltimes given a velocity model and a source-receiver geometry. Raypaths are not required since the SA inversion is based on the computation of the traveltimes only. The FD method is based on the solution of the eikonal equation by means of finite-differences. Once a model parameterization has been chosen, the velocity field is sampled over an equally spaced grid of $N_{x} \times N_{z}$ square cells, $v_{i j}=v\left(x_{i}, z_{j}\right)$, where $x_{i}=x_{\min }+(i-1) h, i=1, \cdots, N_{x}$, and $z_{i}=z_{m i n}+(j-1) h$, $j=1, \cdots, N_{z}$, and

$$
v(x, z)=v_{b}(x, z)+v_{a}(x, z)
$$

The "sampling" process is repeated at each iteration after the corresponding parameters have been updated by the SA algorithm. 


\section{Inverse modeling}

The traveltime inversion problem is cast as a nonlinear optimization problem. For this purpose, I define the cost function

$$
\Phi(\mathbf{m})=\frac{1}{N} \sum_{n=1}^{N} w_{n}\left|T_{n}^{o}-T_{n}^{c}(\mathbf{m})\right|^{q}
$$

where $w_{n}$ are weights, and $N$ is the number of observations. This equation expresses the misfit between the observed and calculated traveltimes. In general, $q=2$ and $w_{n}=1$, which leads to a standard least-squares optimization. But other values for $q$ and $w_{n}$ can also be used. The objective is to minimize equation (12) with respect to $\mathbf{m}$, such that

$$
\Phi(\mathbf{m}) \leq \Phi_{t o l}=\chi^{q}
$$

where $\Phi_{t o l}$ is a tolerance cost associated with the observational errors, and $\chi$ is the expected misfit. Note that $\chi$ has the same units as traveltimes. In general, an estimate of the right-hand side of equation (13) is available, so $\chi$ is a measure of the goodness-of-fit of the model to the observed data.

In addition to minimizing $\Phi(\mathbf{m})$, I specify a set of bounding constraints of the form

$$
A_{k} \leq m_{k} \leq B_{k}, \quad k=1, \cdots, K
$$

This is to avoid undesirable models that may lead to erroneous velocity fields (e.g. negative velocity values). Also, they may be used to specify some prior geophysical knowledge about the underlying model (e.g. an approximate location of the anomaly), and to "freeze" a certain model parameter by setting $A_{k}=B_{k}$, for some $k$, in case it is known by other means.

Due to the severe nonlinearities introduced by the selected parameterization, gradient-based techniques for minimizing equation (12) are prone to converge to a local minimum. This fact can be visualized by considering parameters such as $x_{c}$ and $z_{c}$. By slighting changing one of those parameters, some raypaths may or may not traverse the anomaly. This effect might produce abrupt changes and/or discontinuities in the cost function, specially when the borders of the anomaly are sharp. So, instead of using gradient-based methods, I carry out the minimization of $\Phi(\mathbf{m})$ by means of VFSA. VFSA, an improved SA method, is a versatile and efficient method for finding the global minimum of arbitrary nonlinear objective functions and it only requires one to solve the forward problem at each iteration. Details of the method will not be given here, and the reader is referred to (Ingber, 1989)

\section{$5 \quad$ Examples}

Three models representing various buried structures were considered (refer to Figure 2). Model 1 and Model 3 illustrate anomaly bodies with sharp velocity contrasts, while Model 2 represents a smooth anomaly body. Traveltimes are generated using the FD method over a $100 \times 100$ grid. Distances are given in meters, traveltimes in milliseconds, and velocities in kilometers per second. The acquisition geometry consists of several sources and receivers distributed uniformly along the surface and two vertical boreholes. Both crosswell and well-to-surface (VSP) data were collected using: (1) 5 sources in each well and 5 receivers on the surface (VSP data), and (2) 5 sources in the left well and 5 receivers in the right well (crosswell data).

In the simulations below, data are contaminated with uniform random noise with zero mean and amplitude $\pm b$, where $b$ is a percentage of the maximum observed traveltime. Assuming all weights are equal to one in equation (12), the expected misfit reduces to $\chi=b /(q+1)^{1 / q}$. For $q=2, \chi=b / \sqrt{3}$. In the inversion, the SA iteration stopped when the cost function reached the expected misfit (13), or after a maximum number of iterations (3000 by default). Each iteration involves the solution of the forward problem once. Initial parameter values are selected at random from the specified search limits. For simplicity, the velocity gradients $g_{x}$ and $g_{z}$ are set equal to zero in all cases.

For illustrative purposes only, SA solutions are compared with linearized (LIN) solutions (Aldridge and Oldenburg, 1993). This approach utilizes a standard cell parameterization to represent the model. Though this representation is able to accommodate a larger class of velocity structures than the scheme proposed here, the smoothness imposed by the regularization (to alleviate the nonuniqueness and instability of the inverse problem) favors smooth solutions. 

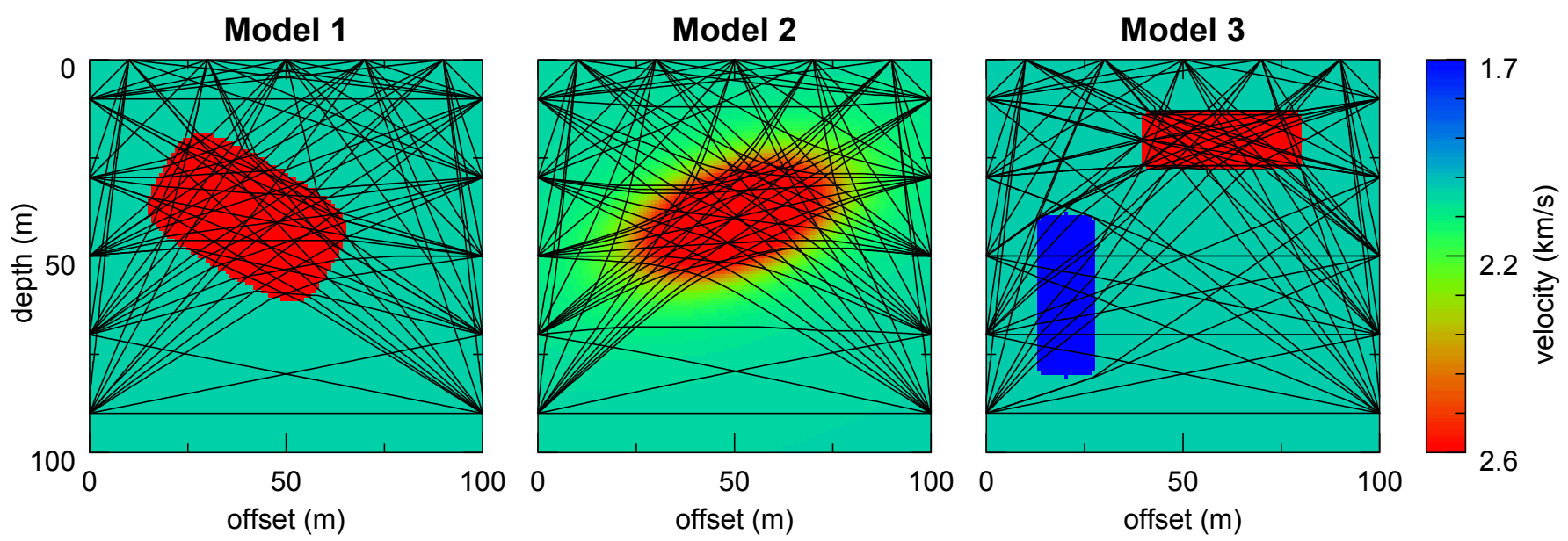

Figure 2: Anomaly models (and raypath coverage) used in the traveltime inversion.

\begin{tabular}{|c|c|c|c|c|c|}
\hline & \multicolumn{2}{|c|}{ Model 1} & \multicolumn{2}{|c|}{ Model 2} & \\
\hline Parameter & True & Mean & True & Mean & Search range \\
\hline$A(\mathrm{~km} / \mathrm{s})$ & 0.5 & $0.5 \pm 0.0$ & 0.5 & $0.5 \pm 0.0$ & $0-1$ \\
\hline$x_{c}(\mathrm{~m})$ & 40.0 & $40.5 \pm 1.2$ & 50.0 & $50.7 \pm 0.6$ & $10-90$ \\
\hline$z_{c}(\mathrm{~m})$ & 40.0 & $40.0 \pm 0.5$ & 40.0 & $39.7 \pm 0.6$ & $10-90$ \\
\hline$R_{x}(\mathrm{~m})$ & 15.0 & $11.3 \pm 2.1$ & 20.0 & $22.4 \pm 2.0$ & $0-50$ \\
\hline$R_{z}(\mathrm{~m})$ & 25.0 & $19.0 \pm 3.7$ & 10.0 & $10.8 \pm 1.2$ & $0-50$ \\
\hline$p$ & 4.0 & $5.3 \pm 1.9$ & 2.0 & $2.4 \pm 0.3$ & $1-10$ \\
\hline$\rho$ & 0.0 & $0.3 \pm 0.2$ & 0.5 & $0.3 \pm 0.1$ & $0-1$ \\
\hline$\theta$ (deg) & 60.0 & $63.0 \pm 4.7$ & 20.0 & $18.8 \pm 3.7$ & $0-90$ \\
\hline$v_{0}(\mathrm{~km} / \mathrm{s})$ & 2.0 & $2.0 \pm 0.0$ & 2.0 & $2.0 \pm 0.0$ & $1-3$ \\
\hline
\end{tabular}

Table 1: Estimated model parameters after 3000 iterations in Models 1 and 2 (10 runs).

\section{Model 1}

Model 1 illustrates a high contrasting anomaly model that sits on a constant velocity background field (see Figure 2). The velocity of the anomaly is $2.5 \mathrm{~km} / \mathrm{s}$ and the background velocity is $2.0 \mathrm{~km} / \mathrm{s}$. Traveltimes were then contaminated with $1 \%$ random noise $(b= \pm 0.61)$, which yields $\chi \simeq 0.35 \mathrm{~ms}$. Figure 3 shows the inversion using the SA approach (10 independent realizations ${ }^{2}$ ), and Table 1 summarizes the results. In general, the expected misfit was achieved after 2000-3000 iterations, as shown in Figure 4. It can be seen that the true model shape and size were recovered quite accurately in most cases. The figure also depicts the inversion using the linearizing approach. The LIN solution achieved the expected misfit, too, but the resulting model is smoother than the SA solution. Due to the low raypath coverage, the shape and size of the anomaly was not recovered as accurately as in the SA case. This can be better appreciated by inspecting the error models which are also depicted in Figure 3. Red areas (errors equal or larger than $10 \%$ ) dominate a large part of the LIN error model. These models were computed by

$$
\text { error model }=\frac{\text { true model }- \text { recovered model }}{\text { true model }} \times 100 \% .
$$

\section{Model 2}

Model 2 represents a smooth elliptical body embedded in a constant background velocity (Figure 2). The background velocity is $2.0 \mathrm{~km} / \mathrm{s}$, and the maximum velocity of the anomaly body is $2.5 \mathrm{~km} / \mathrm{s}$. The results of the inversion are shown

\footnotetext{
${ }^{2}$ Independent realizations were obtained by feeding the random number generator that governs the SA process with different seeds.
} 

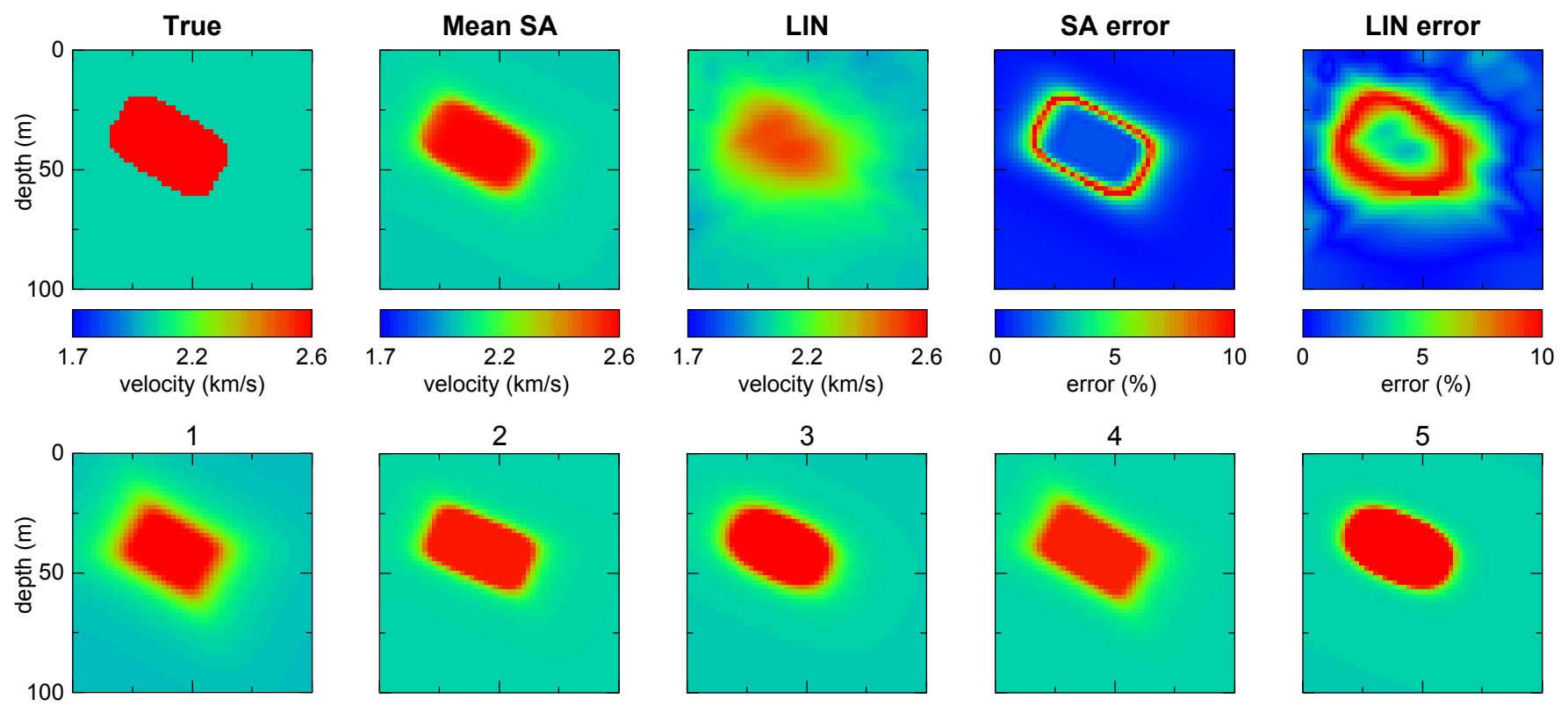

\section{3}

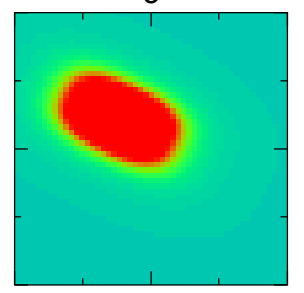

4
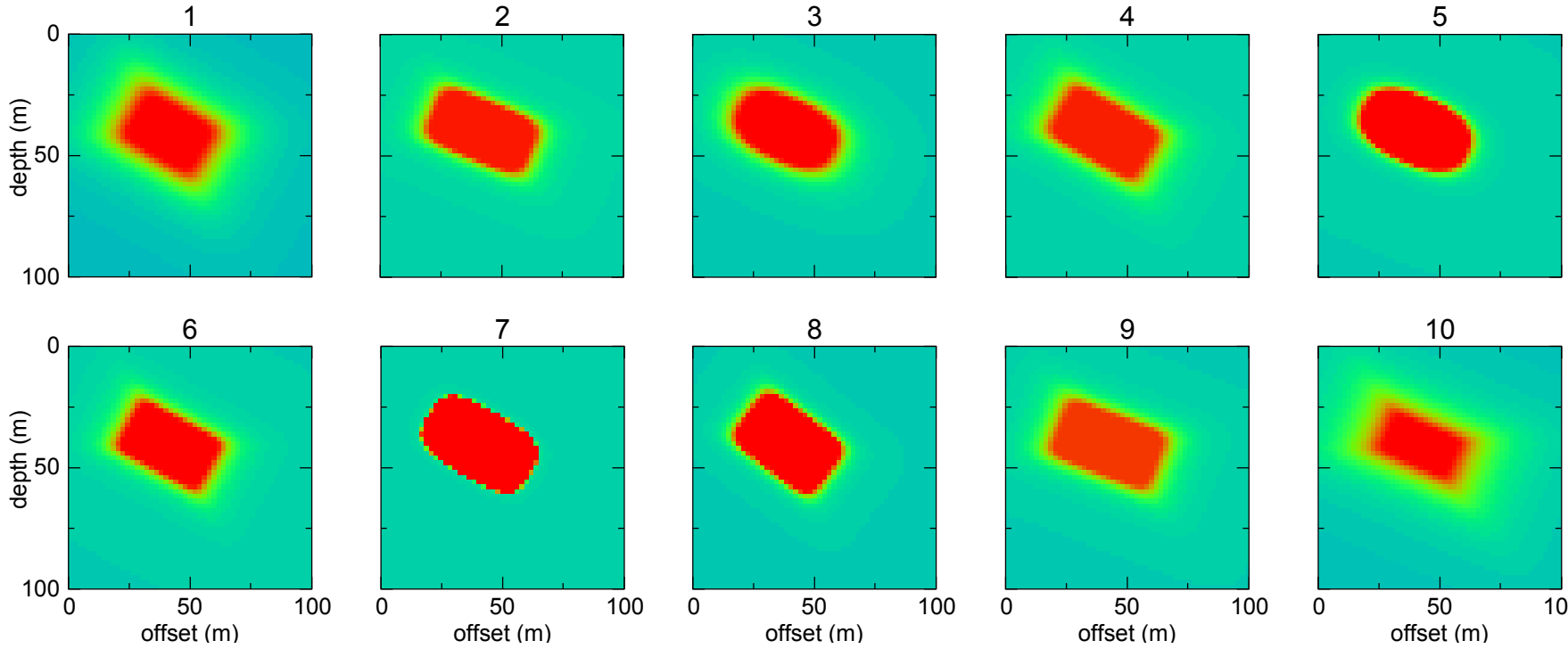

8

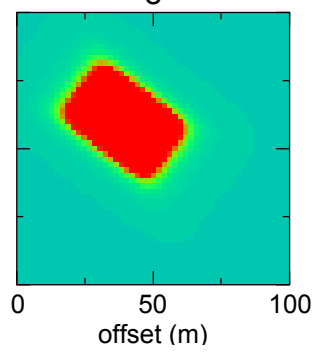

9

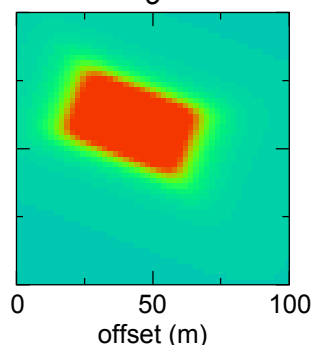

10

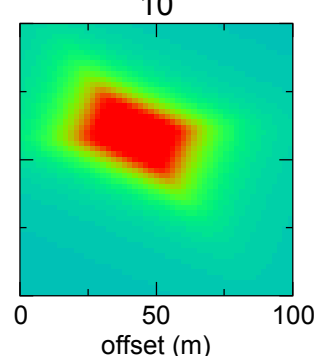

Figure 3: Traveltime inversion in Model 1. The numbered panels show 10 independent SA inversions.

Model 1

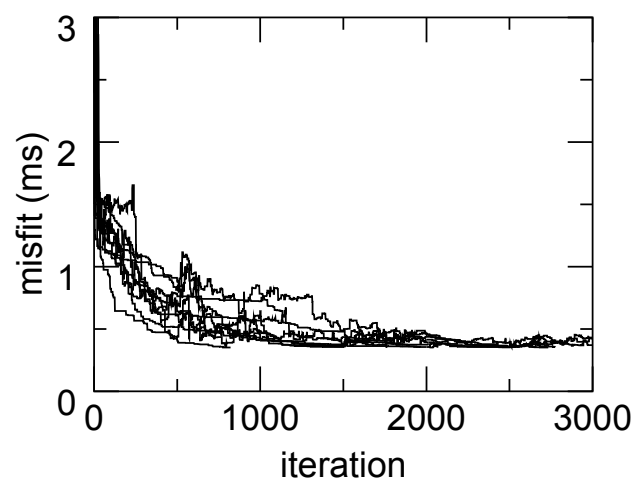

Model 2

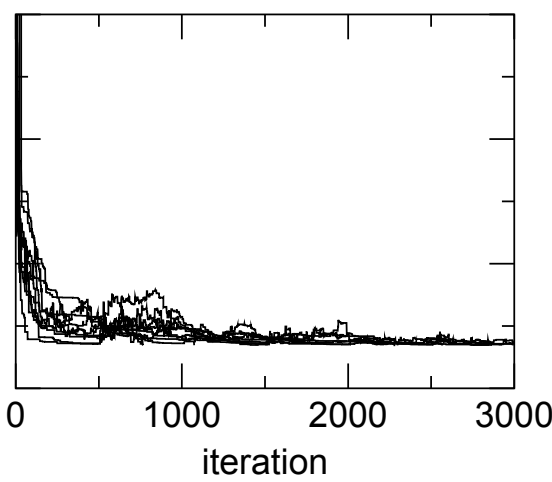

Model 3

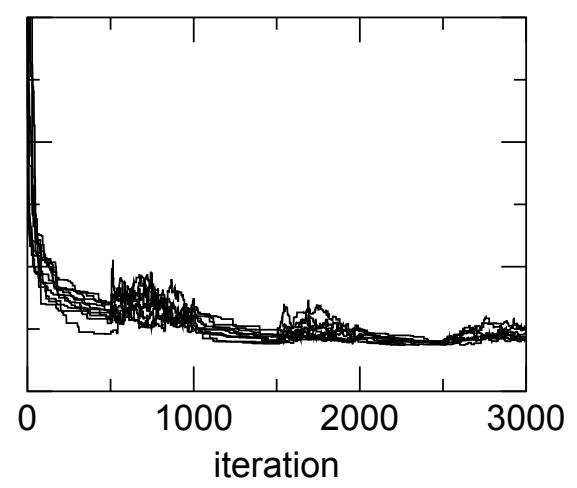

Figure 4: VFSA convergence after 3000 iterations (10 independent realizations). 


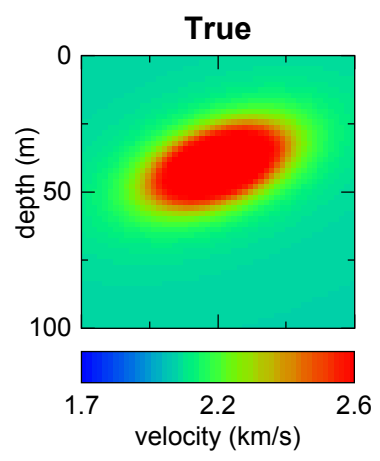

1

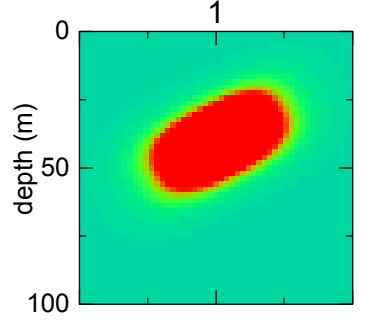

6

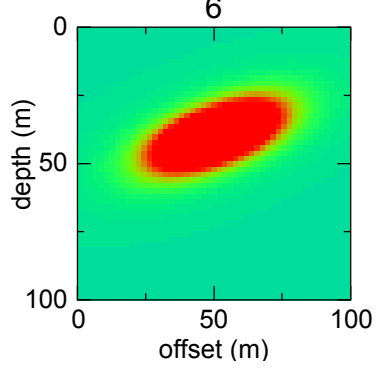

Mean SA

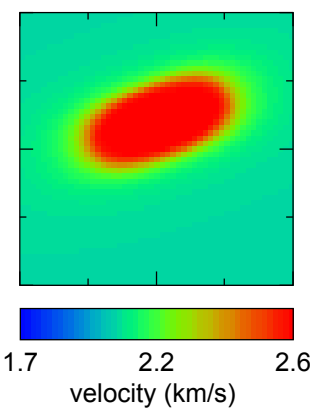

2

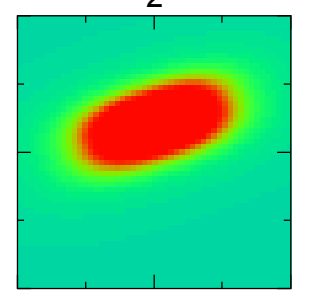

7

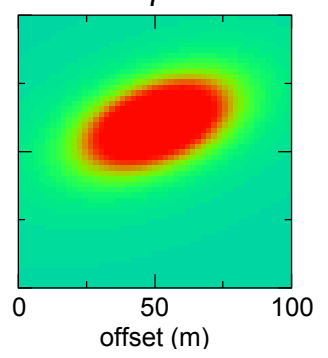

LIN

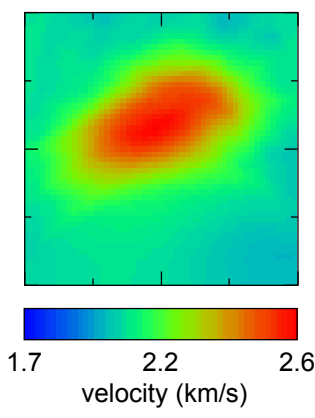

3

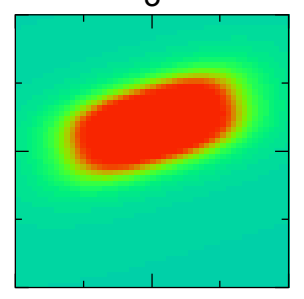

8

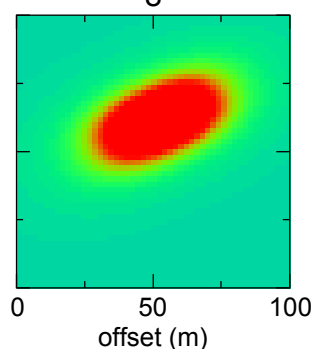

SA error

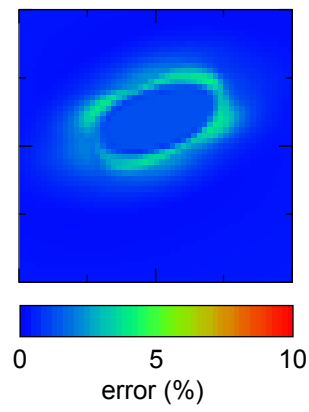

4

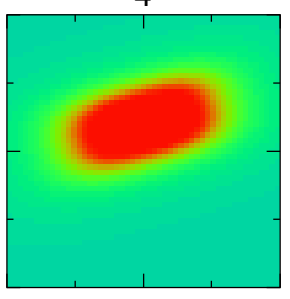

9

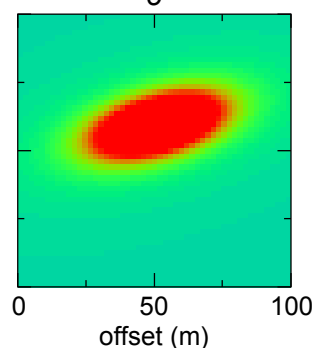

LIN error

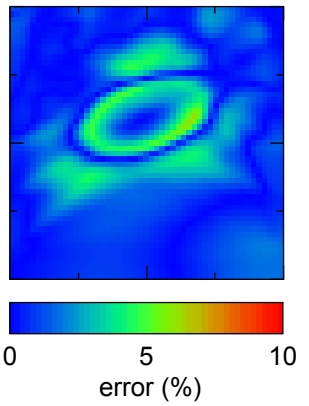

5

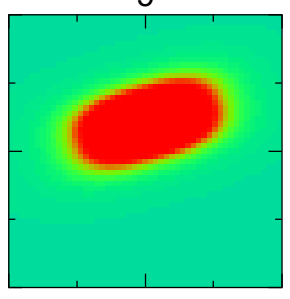

10

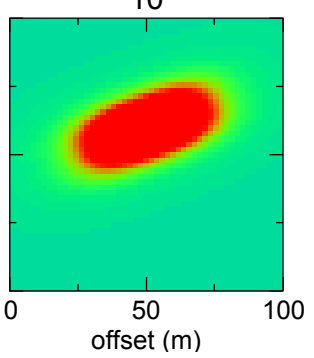

Figure 5: Traveltime inversion in Model 2. The numbered panels show 10 independent SA inversions.

in subsequent panels of Figure 5 and summarized in Table 1. The random noise has $b=0.60 \mathrm{~ms}$, which corresponds to an expected misfit $\chi \simeq 0.35 \mathrm{~ms}$. When compared to the LIN inversion, both results are in good agreement with the true model, though the SA solution appears to be somehow smoother and closer to the original model.

\section{Model 3}

In this experiment, two objects with sharp velocity contrasts were embedded in a constant background velocity of 2.0 $\mathrm{km} / \mathrm{s}$, as shown in Figure 2. The velocities of the structures are $1.75 \mathrm{~km} / \mathrm{s}$ and $2.5 \mathrm{~km} / \mathrm{s}$. Here, the random noise has $b=0.64 \mathrm{~ms}(\chi \simeq 0.37 \mathrm{~ms})$. The results of the inversion using SA after 3000 iterations are shown in Figure 6 and Table 2. Since the LIN approach imposes smoothness for stabilizing the inversion, the reconstructed model is not as accurate as the SA solution. The spreading around the low velocity object is evident due to the poor raypath coverage in this area.

In the previous examples, the number of buried objects was known a priori. In practice, one might guess this number from observing the LIN solution. When there is no clue about how many buried structures to look for, a simple strategy would be to perform successive runs seeking for an increasing number of anomaly structures. When the expected misfit is achieved, the solution obtained so far might be the correct. The idea is to search for the least number of objects that fit the data. Clearly, the issue of looking for a wrong number of buried objects needs further investigation.

\section{Conclusions}

I have demonstrated the ability of the described traveltime tomography procedure for imaging anomaly structures. The traveltime inverse problem is cast as a constrained nonlinear optimization problem, which is solved by means of VFSA 


\begin{tabular}{lrrrrr}
\hline \hline \multicolumn{2}{c}{ Body $\# 1$} & \multicolumn{3}{c}{ Body \#2 } \\
\hline Parameter & True & \multicolumn{1}{c}{ Mean } & True & Mean & Search range \\
\hline$A(\mathrm{~km} / \mathrm{s})$ & 0.5 & $0.5 \pm 0.0$ & -0.25 & $-0.16 \pm 0.1$ & $0-1 /-1-+1$ \\
$x_{c}(\mathrm{~m})$ & 60.0 & $61.2 \pm 0.6$ & 20.0 & $23.6 \pm 3.7$ & $50-80 / 10-50$ \\
$z_{c}(\mathrm{~m})$ & 20.0 & $20.1 \pm 0.3$ & 60.0 & $61.1 \pm 2.2$ & $10-50 / 50-80$ \\
$R_{x}(\mathrm{~m})$ & 7.5 & $5.9 \pm 0.7$ & 7.5 & $12.2 \pm 4.2$ & $5-30$ \\
$R_{z}(\mathrm{~m})$ & 21.0 & $17.7 \pm 2.1$ & 21.0 & $20.6 \pm 2.9$ & $5-30$ \\
$p$ & 10.0 & $7.1 \pm 2.7$ & 10.0 & $6.2 \pm 3.1$ & $2-12$ \\
$\rho$ & 0.0 & $0.1 \pm 0.1$ & 0.5 & $0.3 \pm 0.1$ & $0-0.5$ \\
$\theta(\mathrm{deg})$ & 90.0 & $89.9 \pm 1.7$ & 0.0 & $-8.6 \pm 28.4$ & $-90-+90$ \\
$v_{0}(\mathrm{~km} / \mathrm{s})$ & 2.0 & $2.0 \pm 0.0$ & - & - & $1-3$ \\
\hline \hline
\end{tabular}

Table 2: Estimated model parameters after 3000 iterations in Model 3 (10 runs).

in an attempt to find the global minimum regardless the initial model. The results of the inversion using synthetic data are in very good agreement with the original models. The main drawback of the traveltime inversion problem presented in this work relies perhaps in the fact that it is a time consuming process. As compared to linearizing methods, which usually require just a few iterations (3-10), the SA approach requires a much larger number (1500-3000). For the size of the models used here, this represented a few minutes in a Sun Ultra 1 workstation. The methodology can be readily extended to deal with 3 -D models.

\section{Acknowledgment}

This research was partially funded by the Consortium for the Development of Specialized Seismic Techniques (CDSST), University of British Columbia, Canada. I acknowledge support from Facultad de Ciencias Astronómicas y Geofísicas (Universidad Nacional de La Plata), Agencia Nacional de Promoción Científica y Tecnológica (BID 802/OC-AR) and Consejo Nacional de Investigaciones Científicas y Técnicas (PIP 0363/98), Argentina.

\section{References}

Aldridge, D., and Oldenburg, D., 1993, Two dimensional tomography inversion with finite-difference traveltimes: J. Seism. Expl., 2, 257-274.

Böhm, G., and Vesnaver, A., 1999, In quest of the grid: Geophysics, 64, no. 4, 1116-1125.

Böhm, G., Rossi, G., and Vesnaver, A., 1997, 3-D adaptive tomography by Voronoi polygons: 67th Ann. Internat. Mtg., Soc. Expl. Geophys., Expanded Abstracts, 2019-2022.

Bois, P., Porte, M. L., Lavergne, M., and Thomas, G., 1972, Well-to-well seismic measurements: Geophysics, 37, no. $3,471-480$.

Bregman, N., Bailey, R., and Chapman, C., 1989, Crosshole seismic tomography: Geophysics, 54, 200-215.

Chunduru, R., Sen, M., Stoffa, P., and Nagendra, R., 1995, Non-linear inversion of resistivity profiling data form some regular geometrical bodies: Geophys. Prosp., 43, 979-1003.

Ingber, L., 1989, Very fast simulated re-annealing: Mathl. Comput. Modelling, 12, 967-973.

Michelena, R., and Harris, J., 1991, Tomographic traveltime inversion using natural pixels: Geophysics, 56, no. 5, $635-644$.

Michelini, A., 1995, An adaptive-grid formalism for traveltime tomography: Geophys. J. Int., 121, 489-510.

Nolet, G., Ed., 1987, Seismic tomography with applications in global seismology and exploration geophysics D. Reidel, Dordretcht, The Netherlands. 

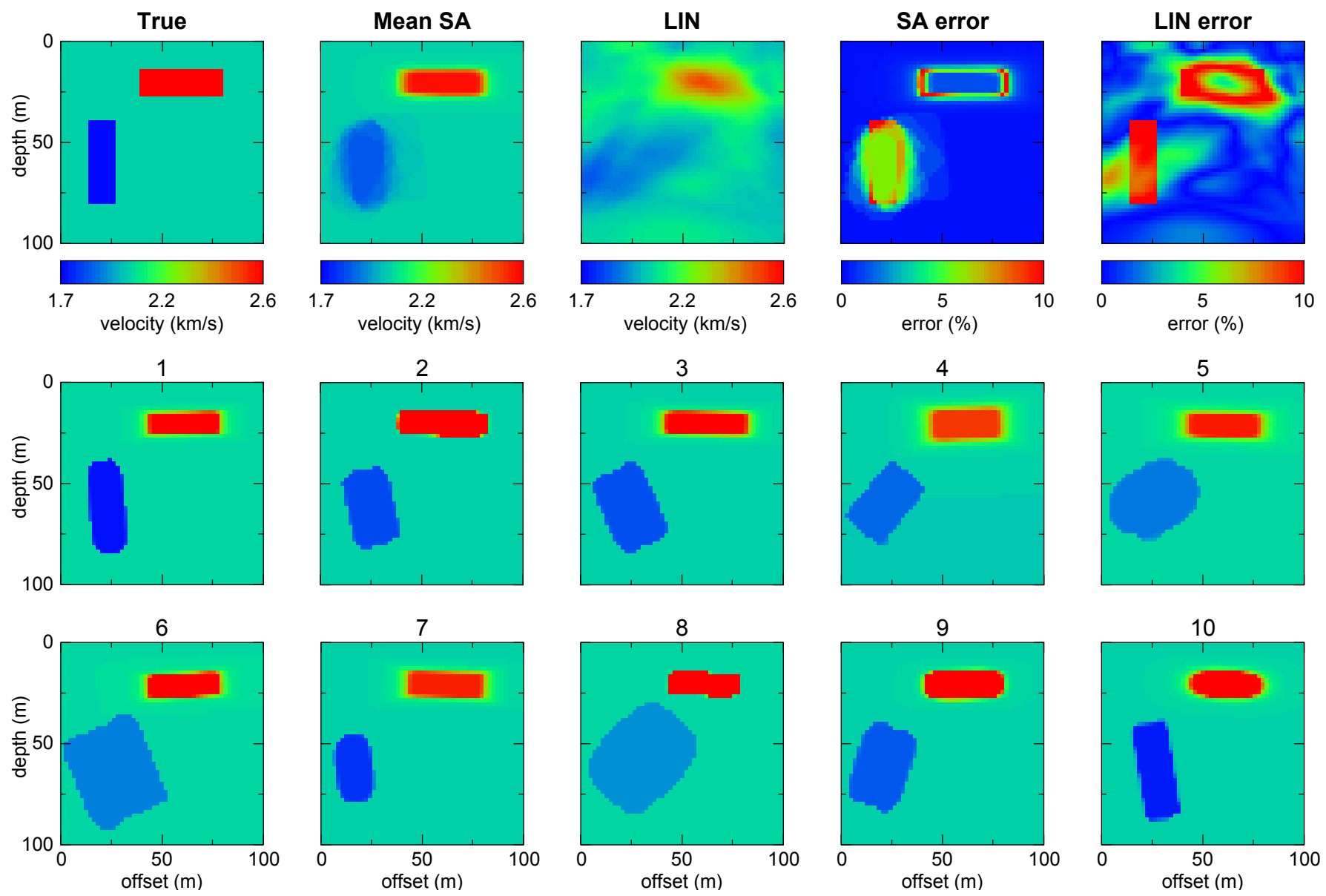

Figure 6: Traveltime inversion in Model 3. The numbered panels show 10 independent SA inversions.

Sen, M., Bhattacharya, B., and Stoffa, P., 1993, Nonlinear inversion of resistivity sounding data: Geophysics, 58, 496-507.

Velis, D. R., and Ulrych, T. J., 1995, Traveltime tomography using very fast simulated annealing: 65th Annual Internat. Mtg., Soc. Expl. Geophys., Expanded Abstracts, 1055-1057.

Velis, D. R., 1998a, Application of simulated annealing to some seismic problems: Ph.D. thesis, University of British Columbia, Vancouver, Canada.

1998b, Nonlinear traveltime inversion: a parametric approach: 68th Ann. Internat. Mtg., Soc. Expl. Geophys., Expanded Abstracts, 1720-1723.

Vesnaver, A., 1994, Towards the uniqueness of tomographic inversion solutions: J. Seis. Expl., 3, 323-334.

Vidale, J., 1988, Finite-difference calculation of traveltimes: Bull. Seis. Soc. Am., 78, 2062-2076. 